\title{
ON ALMOST CONTACT METRIC COMPOUND STRUCTURE
}

\author{
By Yoshiniro TASHiro ANd In-BAE Kim
}

Introduction. K. Yano and U.-H. Ki [8] have recently introduced the notion of ( $f, g, u, v, w, \lambda, \mu, \nu$ )-structure in an odd-dimensional manifold $M$, which is an abstraction of the induced structure in a submanifold of codimension 3 in an almost Hermitian manifold, and studied conditions for such a structure to define an almost contact structure in $M$ and properties of pseudo-umbilical submanifold of codimension 3 satisfying the conditions in a Euclidean space of even-dimension.

In the present paper, we shall introduce in $\S 1$ the notion of metric compound structure in a manifold $M$ of dimension $m$, which is a generalization of $(f, g, u, v, w, \lambda, \mu, \nu)$ and naturally induced in $M$ if $M$ is a submanifold in an almost Hermitian manifold $\tilde{M}$ of dimension $n$. In $\S 2$, we shall seek for conditions in order that a metric compound structure defines an almost contact metric structure in $M$. After the definition of normality in $\S 3$, we shall consider in $\S 4$ submanifolds having a normal contact metric compound structure in a Kaehlerian manifold. In $\S 5$, we shall disscuss properties and give geometrical characterization of pseudo-umbilical submanifolds in a Euclidean space. In $\S 6$, we shall show that a metric compound structure possessing another property gives an almost contact metric structure.

Throughout this paper, we put $l=n-m$ and indices run the following ranges respectively :

$$
\begin{aligned}
& \kappa, \lambda, \mu, \nu, \cdots=1,2, \cdots \cdots \cdots \cdots \cdots \cdots \cdots \cdots \cdots, n \text {; } \\
& h, i, \jmath, k, \cdots=1,2, \cdots, m \text {; } \\
& p, q, r, s, \cdots=\quad m+1, m+2, \cdots, n \text {; } \\
& A, B, C, D, \cdots=1,2, \cdots, m, m+1, \cdots \cdots \cdots, n \text {. }
\end{aligned}
$$

\section{$\S 1$. Metric compound structure}

Let $\tilde{M}$ be an $n$-dimensional almost Hermitian manifold and $(G, \tilde{F})$ the almost Hermitian structure, where $G$ is the almost Hermitian metric and $\tilde{F}$ the almost complex structure of $\tilde{M}$. We denote by $G_{\lambda \mu}$ and $\widetilde{F}_{\lambda^{k}}$ components of $G$ and $\widetilde{F}$ with respect to a local coordinate system $\left(x^{\kappa}\right)$. If $I=\left(\delta_{\lambda_{k}}{ }^{\kappa}\right)$ indicates the identity

Received April 24, 1980. 
tensor, then the structure satisfy the equations

$$
\tilde{F}^{2}=-I ; \tilde{F}_{\mu}{ }^{\lambda} \tilde{F}_{\lambda}{ }^{\kappa}=-\delta_{\mu}{ }^{k}
$$

and

$$
{ }^{t} \tilde{F} G \tilde{F}=G ; \tilde{F}_{\nu}{ }^{\lambda} \tilde{F}_{\mu}{ }^{k} G_{\lambda x}=G_{\nu \mu} .
$$

If we put the covariant components of $\tilde{F}$ as

$$
\tilde{F}^{*}=G \tilde{F} ; \tilde{F}_{\mu \lambda}=\tilde{F}_{\mu}{ }^{k} G_{\lambda \kappa},
$$

then $\tilde{F}_{\mu \lambda}$ is skew-symmetric in $\lambda$ and $\mu$.

Let $M$ be an $m$-dimensional Riemannian manifold and suppose now that it is immersed isometrically in $\tilde{M}$ by the parametric equations

$$
x^{\kappa}=x^{\kappa}\left(y^{h}\right)
$$

by use of a local coordinate system $\left(y^{h}\right)$ of $M$.

We put

$$
B_{i}{ }^{k}=\partial_{i} x^{k}
$$

and denote by $C_{q}{ }^{k} l$ mutually orthogonal unit normal vector fields of $M$. Then the $n$ vectors $B_{\imath}{ }^{k}$ and $C_{q}{ }^{k}$ span the tangent space $T(\tilde{M})$ of $\tilde{M}$ at every point of $M$ and the matrix

$$
B=\left(B_{B}{ }^{\kappa}\right)=\left(B_{\imath}{ }^{\kappa}, C_{q}{ }^{\kappa}\right)
$$

is regular. The metric tensor $g$ of $M$ is related with $G$ of $\tilde{M}$ by

$$
g_{j i}=G_{\mu \lambda} B_{j}^{\mu} B_{\imath}^{\lambda} .
$$

Denoting the contravariant components of $g$ by $g^{i h}$, we put

$$
\begin{aligned}
& B_{\lambda}^{h}=g^{i \hbar} G_{\lambda \kappa} B_{\imath}{ }^{k}, \\
& C_{q \lambda}=G_{\lambda k} C_{q}{ }^{\kappa} .
\end{aligned}
$$

Then the inverse matrix $B^{-1}$ of $B$ is given by

Now we put

$$
B^{-1}=\left(B_{A \lambda}\right)=\left(\begin{array}{l}
B_{\lambda}^{h} \\
C_{p \lambda}
\end{array}\right) \text {. }
$$

$$
F=B^{-1} \tilde{F} B ;\left(F_{B}{ }^{A}\right)=\left(B_{B}{ }^{\lambda} \tilde{F}_{\lambda^{k}} B^{A}{ }_{\kappa}\right)=\left(\begin{array}{cc}
f_{\imath}{ }^{h} & -v_{q}{ }^{h} \\
v_{p \imath} & f_{q p}
\end{array}\right) .
$$

Then the components of four kinds of $F$ are given by

$$
f_{\imath}{ }^{h}=B_{\imath}{ }^{\lambda} \widetilde{F}_{\lambda}{ }^{\kappa} B^{h}{ }_{\kappa}, \quad v_{q}{ }^{h}=-C_{q}{ }^{\lambda} \tilde{F}_{\lambda}{ }^{\kappa} B^{h}{ }_{\kappa},
$$




$$
v_{p \imath}=B_{\imath}{ }^{\lambda} \tilde{F}_{\lambda}{ }^{\kappa} C_{p \kappa}, \quad f_{q p}=C_{q}{ }^{\lambda} \tilde{F}_{\lambda}{ }^{\kappa} C_{p \kappa} .
$$

Since $\tilde{F}^{*}=\left(\tilde{F}_{\mu \lambda}\right)$ is skew-symmetric, we have the relations

$$
v_{p \imath}=v_{p}{ }^{h} g_{i h}
$$

and see that

$$
f_{\jmath i}=B_{\jmath}{ }^{\lambda} B_{\imath}{ }^{\kappa} \tilde{F}_{\lambda \kappa}
$$

is skew-symmetric in $\iota$ and $\jmath$, and

$$
f_{q p}=C_{q}{ }^{\lambda} C_{p}{ }^{\kappa} \tilde{F}_{\lambda \kappa}
$$

is also skew-symmetric in $p$ and $q$. Thus the sets $f=\left(f_{\imath}{ }^{h}\right), v=\left(v_{q}{ }^{h}\right)$ and $f^{\perp}=$ $\left(f_{q p}\right)$ compose a $(1,1)$-tensor, $m$ vector fields and $l(l-1) / 2$ scalar fields on $M$ respectively.

The transforms of the tangent vectors $B_{\imath}{ }^{\kappa}$ and the normal vectors $C_{q}{ }^{\kappa}$ to $M$ by $\widetilde{F}$ are expressed in the form

$$
\widetilde{F}_{\lambda}{ }^{\kappa} B_{\imath}{ }^{\lambda}=f_{\imath}{ }^{h} B_{h}{ }^{\kappa}+v_{p i} C_{p}{ }^{\kappa}
$$

and

$$
\widetilde{F}_{\lambda}{ }^{k} C_{q}{ }^{\lambda}=-v_{q}{ }^{h} B_{h}{ }^{k}+f_{q p} C_{p}{ }^{k},
$$

where and in the sequel summation convention is also applied to repeated lower indices $p, q, r, \cdots$ on their own range $m+1, m+2, \cdots, n$. Since the matrix (1.7) satisfies the equation

$$
F^{2}=-I \text {, }
$$

the quantities $f, v$ and $f^{\perp}$ are in the relation

$$
\begin{gathered}
f_{\jmath}{ }^{\imath} f_{\imath}{ }^{h}=-\delta_{\jmath}{ }^{h}+v_{q \jmath} v_{q}{ }^{h}, \\
f_{\jmath}{ }^{\imath} v_{p \imath}=-v_{q \jmath} f_{q p}=f_{p q} v_{q \jmath}, \\
v_{r}^{\imath} f_{\imath}{ }^{h}=-f_{r q} v_{q}{ }^{h}, \\
f_{r q} f_{q p}=-\delta_{r p}+v_{r}{ }^{2} v_{p \imath} .
\end{gathered}
$$

The relation (1.6) is equivalent to

$$
f_{j}{ }^{k} f_{\imath}{ }^{h} g_{k h}+v_{q j} v_{q \imath}=g_{j i} \text {. }
$$

Now removing the almost Hermitian ambient manifold $\tilde{M}$, we consider an $m$-dimensional Riemannian manifold $M$ admitting a metric tensor $g$, a $(1,1)$-tensor field $f, m$ vector fields $v_{q}$ and $l(l-1) / 2$ scalar fields $f_{q p}$ such that they satisfy the relations (1.13), (1.14), (1.15), (1.16) and (1.17), and call the totality $\left(f, g, v, f^{\perp}\right)$ of these quantities a metric compound structure on $M$.

If we put 


$$
\tilde{F}=\left(\begin{array}{cc}
f_{\imath}{ }^{h} & -v_{q}{ }^{h} \\
v_{p \imath} & f_{q p}
\end{array}\right) \text { and } G=\left(\begin{array}{cc}
g_{j i} & 0 \\
0 & \delta_{q p}
\end{array}\right),
$$

then the set $(\tilde{F}, G)$ defines an almost Hermitian structure in the product space $M \times R^{l}$ of the manifold $M$ with an $l$-dimensional Euclidean space $R^{l}$.

\section{$\S 2$. Almost contact metric compound structure}

We shall suppose that the tensor field $f$ together with the metric tensor $g$, a contravariant vector field $\xi=\left(\xi^{h}\right)$ and a covariant vector field $\eta=\left(\eta_{2}\right)$ compose an almost contact metric structure on $M$. Then we have

$$
\begin{gathered}
f_{j}{ }^{2} f_{\imath}{ }^{h}=-\delta_{j}{ }^{h}+\eta_{j} \xi^{h}, \\
f_{\imath}{ }^{h} \xi^{2}=0, \quad f_{\imath}{ }^{h} \eta_{h}=0, \\
\xi^{2} \eta_{\imath}=1
\end{gathered}
$$

and

$$
f_{j}{ }^{k} f_{\imath}{ }^{n} g_{k h}+\eta_{j} \eta_{\imath}=g_{j i} .
$$

In this case we know that the dimension $m$ of $M$ is odd and the rank of $f=$ $\left(f_{0}{ }^{i}\right)$ is equal to $m-1$.

Comparing (1.17) with (2.4), we have

$$
v_{q j} v_{q 2}=\eta_{j} \eta_{\imath} .
$$

This equation shows that the product of the matrix $\left(v_{q 2}\right)$ with the transpose is of rank 1 and consequently that the matrix $\left(v_{q 2}\right)$ by itself is of rank 1 . Therefore we may put

$$
v_{q 2}=\nu_{q} \eta_{\imath},
$$

where $\nu_{q}$ are proportional factors. Since $v_{q i} v_{q}{ }^{2}=\eta_{i} \xi^{2}=1$, We have

$$
\nu_{q} \nu_{q}=1
$$

and the equations (1.15) and (1.16) are reduced to

$$
f_{q p} \nu_{p}=0
$$

and

$$
f_{r q} f_{q p}=-\delta_{r p}+\nu_{r} \nu_{p}
$$

respectively. The equations (2.7), (2.8) and (2.9) mean that the set $\left(f^{\perp}, g^{\perp}, \nu\right)$ forms an almost contact metric structure on $R^{l}$ at every point of $M$, where $g^{\perp}=\left(\delta_{q p}\right)$, and we see that the dimension $l$ of $R^{l}$ is odd. 
Conversely, starting from the almost contact metric structure $\left(f^{\perp}, g^{\perp}, \nu\right)$ on $R^{l}$ at every point of $M$, we can prove that the metric compound structure $\left(f, g, v, f^{\perp}\right)$ introduces an almost contact metric structure $(f, g, \xi, \eta)$ on $M$. Thus we have

THEOREM 1. Let $\left(f, g, v, f^{\perp}\right)$ be a metric compound structure on $M$. In order that $f$ and $g$ constitute an almost contact metric structure $(f, g, \xi, \eta)$ on $M$, it is necessary and sufficient that $f^{\perp}$ and $g^{\perp}$ constitute an almost contact metric structure $\left(f^{\perp}, g^{\perp}, \nu\right)$ on $R^{l}$ at every point of $M$.

A metric compound structure satisfying the condition in the above theorem is called an almost contact metric compound structure on $M$. In the following we shall confine ourselves to such structures. From the above discussions we can state the following

THEOREM 2. In order that a metric compound structure $\left(f, g, v, f^{\perp}\right)$ is almost contact, it is necessary and sufficient that the matrix $\left(v_{q}^{i}\right)$ is of rank 1 , that is, the $l$ vector fields $v_{q}$ are all parallel to each other.

\section{$\S 3$. The Nijenhuis tensor}

Denoting $\partial_{j}=\partial / \partial y^{\nu}$ and regarding $\partial_{q}$ as null operators, we define the Nijenhuis tensor of the metric compound structure (1.18) in $M \times R^{l}$ by

$$
S_{C B}{ }^{A}=\widetilde{F}_{C}{ }^{E}\left(\partial_{E} \widetilde{F}_{B}{ }^{A}-\partial_{B} \widetilde{F}_{E}{ }^{A}\right)-\widetilde{F}_{B}{ }^{E}\left(\partial_{E} \widetilde{F}_{C}{ }^{A}-\partial_{C} \widetilde{F}_{E}^{A}\right) .
$$

Using (1.18), we can write down $S_{C B}{ }^{A}$ as the followings;

$$
\begin{aligned}
& S_{j i}{ }^{h}=f_{\jmath}^{l}\left(\partial_{l} f_{l}{ }^{h}-\partial_{i} f_{l}{ }^{h}\right)-f_{l}^{l}\left(\partial_{l} f_{\jmath}{ }^{h}-\partial_{\jmath} f_{l}{ }^{h}\right)+v_{\jmath s} \partial_{i} v_{s}{ }^{h}-v_{i s} \partial_{j} v_{s}{ }^{h}, \\
& S_{j i p}=f_{\jmath}^{l}\left(\partial_{l} v_{p i}-\partial_{i} v_{p l}\right)-f_{l}{ }^{l}\left(\partial_{l} v_{p j}-\partial_{\jmath} v_{p l}\right)-v_{\jmath s} \partial_{i} f_{s p}+v_{i s} \partial_{\jmath} f_{s p}, \\
& S_{\jmath q}{ }^{h}=-f_{\jmath}{ }^{l} \partial_{l} v_{q}{ }^{h}+v_{q}^{l}\left(\partial_{l} f_{\jmath}{ }^{h}-\partial_{\jmath} f_{l}{ }^{h}\right)+f_{q s} \partial_{j} v_{s}{ }^{h}, \\
& S_{\jmath q p}=f_{\jmath}{ }^{l} \partial_{l} f_{q p}+v_{q}^{l}\left(\partial_{l} v_{p j}-\partial_{j} v_{p l}\right)+f_{q s} \partial_{\jmath} f_{s p}, \\
& S_{r q}{ }^{h}=v_{r}^{l} \partial_{l} v_{q}{ }^{h}-v_{q}^{l} \partial_{l} v_{r}{ }^{h}, \\
& S_{r q p}=-v_{r}^{l} \partial_{l} f_{q p}+v_{q}^{l} \partial_{l} f_{r p} .
\end{aligned}
$$

If the metric compound structure $\left(f, g, v, f^{\perp}\right)$ gives an almost contact metric structures $(f, g, \xi, \eta)$ on $M$ and $\left(f^{\perp}, g^{\perp}, \nu\right)$ on $R^{l}$, then the above expressions are reduced to

$$
\begin{aligned}
S_{j i}{ }^{h}= & f_{\jmath}{ }^{l}\left(\partial_{l} f_{l}{ }^{h}-\partial_{i} f_{l}{ }^{h}\right)-f_{l}{ }^{l}\left(\partial_{l} f_{\jmath}{ }^{h}-\partial_{\jmath} f_{l}{ }^{h}\right)+\eta_{j} \partial_{i} \xi^{h}-\eta_{i} \partial_{j} \xi^{h}, \\
S_{j i p}= & {\left[f_{j}{ }^{l}\left(\partial_{l} \eta_{i}-\partial_{i} \eta_{l}\right)-f_{l}{ }^{l}\left(\partial_{l} \eta_{j}-\partial_{j} \eta_{l}\right)\right] \nu_{p} } \\
& +\left(f_{\jmath}^{l} \eta_{i}-f_{\imath}{ }^{l} \eta_{j}\right) \partial_{l} \nu_{p}+\left(\eta_{j} \partial_{i} \nu_{s}-\eta_{i} \partial_{j} \nu_{s}\right) f_{s p},
\end{aligned}
$$




$$
\begin{aligned}
& S_{\jmath q}^{h}=\left[\xi^{l}\left(\partial_{l} f_{\jmath}^{h}-\partial_{\jmath} f_{l}{ }^{h}\right)-f_{\jmath}{ }^{l} \partial_{l} \xi^{h}\right] \nu_{q}-\left(f_{\jmath}^{l} \partial_{l} \nu_{q}+f_{q s} \partial_{\jmath} \nu_{s}\right) \xi^{h}, \\
& S_{\jmath q p}=\left(\xi^{l} \partial_{l} \eta_{j}-\xi^{l} \partial_{\jmath} \eta_{l}\right) \nu_{q} \nu_{p}+\left(\eta_{\jmath} \xi^{l} \partial_{l} \nu_{p}-\partial_{j} \nu_{p}\right) \nu_{q}+f_{\jmath}{ }^{l} \partial_{l} f_{q p}+f_{q s} \partial_{\jmath} f_{s p}, \\
& S_{r q}{ }^{h}=\left(\nu_{r} \xi^{l} \partial_{l} \nu_{q}-\nu_{q} \xi^{l} \partial_{l} \nu_{r}\right) \xi^{h}, \\
& S_{r q p}=-\nu_{r} \xi^{l} \partial_{l} f_{q p}+\nu_{q} \xi^{l} \partial_{l} f_{r p},
\end{aligned}
$$

because $\nu_{q} \nu_{q}=1$ and $\nu_{q} \partial_{j} \nu_{q}=0$.

On the other hand, the Nijenhuis tensors of the almost contact metric structure $(f, g, \xi, \eta)$ are given by $([4])$

$$
\begin{aligned}
& N_{j i}{ }^{h}=f_{\jmath}{ }^{l}\left(\partial_{l} f_{l}{ }^{h}-\partial_{l} f_{l}{ }^{h}\right)-f_{l}{ }^{l}\left(\partial_{l} f_{\jmath}{ }^{h}-\partial_{\jmath} f_{l}{ }^{h}\right)+\eta_{\jmath} \partial_{l} \xi^{h}-\eta_{i} \partial_{\jmath} \xi^{h}, \\
& N_{j i}=f_{\jmath}{ }^{l}\left(\partial_{l} \eta_{i}-\partial_{i} \eta_{l}\right)-f_{l}{ }^{l}\left(\partial_{l} \eta_{j}-\partial_{j} \eta_{l}\right), \\
& N_{\jmath}{ }^{h}=\xi^{l}\left(\partial_{l} f_{\jmath}{ }^{h}-\partial_{\jmath} f_{l}{ }^{h}\right)-f_{\jmath}{ }^{l} \partial_{l} \xi^{h}, \\
& N_{\jmath}=\xi^{l} \partial_{l} \eta_{j}-\xi^{l} \partial_{j} \eta_{l} .
\end{aligned}
$$

Comparing (3.2) with (3.3), we have the equations

$$
\begin{array}{cl}
N_{\jmath i}{ }^{h}=S_{j i}{ }^{h}, & N_{j i}=S_{j i p} \nu_{p}, \\
N_{\jmath}{ }^{h}=S_{\jmath q}{ }^{h} \nu_{q}, & N_{\jmath}=S_{\jmath q p} \nu_{q} \nu_{p} .
\end{array}
$$

Therefore we obtain, from (3.4), the following

THEOREM 3. Let $\left(f, g, v, f^{\perp}\right)$ be an almost contact metric compound structure on $M$. In order for the almost contact metric structure $(f, g, \xi, \eta)$ on $M$ to be normal, it is necessary and sufficient that $S_{j i}{ }^{h}=0$.

\section{$\S 4$. Submanifolds of codimension $l$ of an almost Hermitian manifold}

In this section we assume that $M$ is an $m$-dimensional submanifold of codimension $l$ of an almost Hermitian manifold $\tilde{M}$ and $C_{p}=\left(C_{p}{ }^{\lambda}\right)$ are mutually orthogonal unit vector normal to $M$ in $\tilde{M}$, that is,

$$
G_{\mu \lambda} C_{q}{ }^{\mu} B_{\imath}{ }^{\lambda}=0, \quad G_{\mu \lambda} C_{q}{ }^{\mu} C_{p}{ }^{\lambda}=g_{q p}=\delta_{q p},
$$

and that the induced metric compound structure $\left(f, g, v, f^{\perp}\right)$ on $M$ from the almost Hermitian structure $(G, \widetilde{F})$ on $\tilde{M}$ defines an almost contact structure. The vector field $N^{\lambda}$ defined by

$$
N^{\lambda}=\nu_{p} C_{p}^{2}
$$

is unit normal to $M$ in $\tilde{M}$ because $G_{\mu \lambda} N^{\mu} N^{\lambda}=1$. The transforms of the tangent vectors $B_{\imath}{ }^{\lambda}$ and the normal vectors $C_{p}{ }^{\lambda}$ by $\tilde{F}$ is given by

$$
\tilde{F}_{\mu}{ }^{\lambda} B_{i}{ }^{\mu}=f_{i}{ }^{n} B_{h}{ }^{\lambda}+\eta_{i} N^{\lambda}
$$


and

$$
\tilde{F}_{\mu}{ }^{\lambda} C_{q}{ }^{\mu}=-\nu_{q} \xi^{n} B_{h}{ }^{2}+f_{q p} C_{p}{ }^{2}
$$

respectively.

It is well-known that the submanifold $M$ of an almost Hermitian manifold satisfying (4.3) is semi-invariant with respect to $N^{\lambda}$ and we call $N^{\lambda}$ the distinguished normal to $M[6]$.

From (4.2) and (4.4) we have

$$
\widetilde{F}_{\mu}^{\lambda} N^{\mu}=-\xi^{h} B_{h}^{\lambda},
$$

and hence the transform of the distinguished normal $N^{\lambda}$ by the almost complex structure $\tilde{F}$ of $\tilde{M}$ is tangent to $M$.

Conversely suppose that the submanifold $M$ of codimension $l$ of the almost Hermitian manifold $\tilde{M}$ is semi-invariant with respect to a unit normal $N^{\lambda}$ whose transform by $\tilde{F}$ is tangent to $M$, then we have (4.3) and (4.5) for a vector $\xi^{h}$ and a 1 -form $\eta_{2}=g_{i h} \xi^{h}$ of $M$. Applying $\tilde{F}$ to (4.3) and (4.5), we obtain

$$
\begin{array}{ll}
f_{\jmath}{ }^{h} f_{h}{ }^{\imath}=-\delta_{\jmath}{ }^{2}+\eta_{\jmath} \xi^{2}, & \eta_{\jmath} f_{i}{ }^{\jmath}=0, \\
f_{\jmath}{ }^{2} \xi^{\jmath}=0, & \eta_{i} \xi^{\imath}=1,
\end{array}
$$

We also have, from (1.2), (1.6) and (4.3),

$$
f_{j}{ }^{k} f_{\imath}{ }^{h} g_{k h}=g_{j i}-\eta_{j} \eta_{\imath} .
$$

Therefore we see that the set $(f, g, \xi, \eta)$ defines an almost contact metric structure. As we have seen in $\S 2$, the induced set $\left(f^{\perp}, g^{\perp}, \nu\right)$ also defines an almost contact metric structure. Then we have

THEOREM 4. In order for an induced metric compound structure $\left(f, g, v, f^{\perp}\right)$ on a submanifold $M$ of codimensional $l$ of an almost Hermitian manifold $\tilde{M}$ to be an almost contact, it is necessary and sufficient that the submanifold $M$ is semiinvariant with respect to a unit normal vector field whose transform by $\tilde{F}$ is tangent to the submanifold.

Now denoting by $\nabla$, the operator of van der Waerden-Bortolotti covariant differentiation with respect to $g_{j i}$, we have the Gauss equation for $M$ in $\tilde{M}$

$$
\nabla_{j} B_{\imath}{ }^{\lambda}=h_{j i p} C_{p}^{\lambda},
$$

where $h_{j i p}$ is the second fundamental tensor with respect to the normal $C_{p}{ }^{\lambda}$. The mean curvature vector is defined by

$$
H^{\lambda}=(1 / m) g^{j i} \nabla_{j} B_{\imath}{ }^{\lambda}=(1 / m) h_{\imath}{ }^{l}{ }_{p} C_{p}{ }^{\lambda},
$$

where $h_{l}{ }^{l} p=g^{j i} h_{j i p}$. The Weingarten equation is given by 


$$
\nabla_{\jmath} C_{p}{ }^{\lambda}=-h_{\rho p}{ }^{2} B_{\imath}{ }^{\lambda}+l_{\rho p q} C_{q}{ }^{\lambda},
$$

where $l_{\jmath p q}$ is the third fundamental tensor. Differentiating (4.1) covariantly and making use of (4.6) and (4.8), we have

$$
\begin{gathered}
h_{\jmath q}{ }^{l} g_{l \imath}=h_{j i q}, \\
l_{\jmath q p}=-l_{\jmath p q} .
\end{gathered}
$$

We put $h_{j i p} \nu_{p}=h_{j p}{ }^{h} g_{i n} \nu_{p}=h_{j i}$ and call $h_{j i}$ the intrinsic second fundamental tensor of $M$. Differentiating $N^{\lambda}$ covariantly and using (4.8), we find

$$
\nabla_{j} N^{\lambda}=-h_{j}{ }^{2} B_{\imath}{ }^{\lambda}+\left(\nabla_{j} \nu_{p}+\nu_{q} l_{j q p}\right) C_{p}{ }^{\lambda} .
$$

Now we assume that the ambient manifold $\tilde{M}$ is Kaehlerian. Differentiating (4.3) covariantly and taking account of (4.4), (4.6), (4.8) and (4.11), we have

$$
\begin{aligned}
h_{j i q}\left(-\nu_{q} \xi^{h} B_{h}{ }^{\lambda}+f_{q p} C_{p}{ }^{\lambda}\right)= & \left(\nabla_{j} f_{\imath}{ }^{h}\right) B_{h}{ }^{\lambda}+f_{\imath}{ }^{h} h_{j h p} C_{p}{ }^{\lambda} \\
& +\left(\nabla_{j} \eta_{\imath}\right) N^{\lambda}+\eta_{i}\left[-h_{j}{ }^{h} B_{h}{ }^{\lambda}+\left(\nabla_{j} \nu_{p}+\nu_{q} l_{j q p}\right) C_{p}{ }^{\lambda}\right],
\end{aligned}
$$

from which follow the equations

$$
\begin{gathered}
\nabla_{j} f_{i}{ }^{h}=-h_{j i} \xi^{h}+\eta_{i} h_{j}{ }^{h}, \\
\left(\nabla_{j} \eta_{\imath}\right) \nu_{p}+\eta_{i}\left(\nabla_{j} \nu_{p}\right)=h_{j i q} f_{q p}-f_{\imath}{ }^{l} h_{j l p}-\eta_{i} \nu_{q} l_{j q p} .
\end{gathered}
$$

Transvecting (4.13) with $\nu_{p}$ and $\xi^{2}$, we obtain

$$
\begin{gathered}
\nabla_{j} \eta_{i}=-h_{j l} f_{\imath}{ }^{l}, \\
\nabla_{j} \nu_{p}=\xi^{l} h_{j l q} f_{q p}-\nu_{q} l_{j q p} .
\end{gathered}
$$

Also, differentiating (4.4) covariantly and taking account of (4.3), (4.4), (4.6) and (4.8), we have

$$
\begin{aligned}
& -h_{\jmath q}{ }^{2}\left(f_{\imath}{ }^{h} B_{h}{ }^{\lambda}+\eta_{i} \nu_{p} C_{p}{ }^{\lambda}\right)+l_{\rho q r}\left(-\nu_{r} \xi^{h} B_{h}{ }^{\lambda}+f_{r p} C_{p}{ }^{\lambda}\right) \\
= & -\left(\nabla_{j}\left(\nu_{q} \xi^{h}\right)\right) B_{h}{ }^{\lambda}-\nu_{q} \xi^{l} h_{j l p} C_{p}{ }^{\lambda}+\left(\nabla_{j} f_{q p}\right) C_{p}{ }^{\lambda}+f_{q r}\left(-h_{\jmath r}{ }^{h} B_{h}{ }^{\lambda}+l_{\jmath r p} C_{p}{ }^{\lambda}\right),
\end{aligned}
$$

from which follow the equations

$$
\begin{gathered}
\left(\nabla_{j} \nu_{q}\right) \xi^{h}+\nu_{q}\left(\nabla_{j} \xi^{h}\right)=h_{\jmath q}{ }^{\imath} f_{\imath}{ }^{h}+l_{\partial q r} \nu_{r} \xi^{h}-h_{\jmath r}{ }^{h} f_{q r}, \\
\nabla_{j} f_{q p}=\nu_{q} \xi^{l} h_{j l p}-\nu_{p} \xi^{l} h_{j l q}+l_{\partial q r} f_{r p}-l_{\jmath p r} f_{r q} .
\end{gathered}
$$

Suppose that the almost contact metric structure $(f, g, \xi, \eta)$ on $M$ is normal, that is,

$$
f_{j}{ }^{l}\left(\nabla_{l} f_{\imath}{ }^{h}-\nabla_{\imath} f_{l}{ }^{h}\right)-f_{\imath}{ }^{l}\left(\nabla_{l} f_{j}{ }^{h}-\nabla_{j} f_{l}{ }^{h}\right)+\eta_{j} \nabla_{i} \xi^{h}-\eta_{i} \nabla_{j} \xi^{h}=0 .
$$

Then, substituting (4.12) and (4.14) into this equation, we have the equation 


$$
\left(f_{\jmath}{ }^{l} h_{l}{ }^{h}-h_{\jmath}{ }^{l} f_{l}{ }^{h}\right) \eta_{\imath}=\left(f_{l}{ }^{l} h_{l}{ }^{h}-h_{\imath}{ }^{l} f_{l}{ }^{h}\right) \eta_{\jmath}
$$

and, transvecting this equation with $\xi^{2}$,

$$
f_{\jmath}{ }^{l} h_{l}{ }^{h}-h_{\jmath}{ }^{l} f_{l}{ }^{h}=-\xi^{2} h_{\imath}{ }^{l} f_{l}{ }^{h} \eta_{\jmath} .
$$

Transvecting (4.18) with $f_{k}{ }^{\jmath}$ and with $f_{h}{ }^{2}$ successively, we have the equations

and

$$
-h_{k}{ }^{h}+\eta_{k} \xi^{l} h_{l}{ }^{h}=f_{k}^{j} h_{\jmath}{ }^{l} f_{l}{ }^{h}
$$

$$
f_{k}{ }^{l} h_{l}{ }^{2}-h_{k}{ }^{l} f_{l}{ }^{2}=\eta_{l} h_{\jmath}{ }^{l} f_{k}{ }^{j} \xi^{2}-\eta_{k} \xi^{l} h_{l}{ }^{h} f_{h}{ }^{2} .
$$

Comparing (4.19) with (4.18), we find $\eta_{l} h_{\jmath}{ }^{l} f_{k}{ }^{j} \xi^{\imath}=0$ or equivalently $\xi^{\imath} h_{\imath}{ }^{l} f_{l}{ }^{\jmath}=0$. Moreover, substituting this equation into (4.18), we have

$$
f_{j}{ }^{l} h_{l}{ }^{h}=h_{\jmath}{ }^{l} f_{l}{ }^{h} .
$$

Thus we have

THEOREM 5. Suppose that the submanifold $M$ of codimension $l$ of a Kaehlerian manifold $\tilde{M}$ admits an almost contact metric compound structure $\left(f, g, v, f^{\perp}\right)$. Then, in order for the almost contact metruc structure $(f, g, \xi, \eta)$ on $M$ to be normal, it is necessary and sufficient that the intrinsic second fundamental tensor $h$ and $f$ commute.

Suppose that the almost contact metric structure $(f, g, \xi, \eta)$ on $M$ is normal contact, that is, it satisfies (4.20) and

$$
\nabla_{j} \eta_{i}-\nabla_{\imath} \eta_{j}=2 f_{j i} .
$$

Then, substituting (4.14) into the equation (4.21), we have

$$
-h_{\jmath l} f_{\imath}{ }^{l}+h_{i l} f_{\jmath}{ }^{l}=2 f_{\jmath i},
$$

from which follows the equation

$$
h_{\jmath}{ }^{l} f_{l}{ }^{h}+f_{\jmath}{ }^{l} h_{l}{ }^{h}=2 f_{\jmath}{ }^{h} .
$$

Substituting (4.20) into this equation, we have

$$
h_{\jmath}^{l} f_{l}^{h}=f_{\jmath}{ }^{n} \text {, }
$$

and, transvecting with $\hat{\xi}^{3}$,

$$
\xi^{\jmath} h_{\jmath}{ }^{l} f_{l}{ }^{h}=0 .
$$

Transvecting this equation with $f_{h}{ }^{2}$, we obtain $\xi^{\jmath} h_{\jmath}{ }^{2}=\alpha \xi^{2}$, where we have put

$$
\alpha=\xi^{j} \xi^{\imath} h_{j \imath} .
$$

Transvecting (4.22) with $f_{h}{ }^{2}$, we have 


$$
h_{\jmath}{ }^{l}\left(-\delta_{l}{ }^{2}+\eta_{l} \xi^{i}\right)=-\delta_{\jmath}{ }^{2}+\eta_{j} \xi^{2}
$$

or equivalently

$$
h_{\jmath 2}=g_{j i}+(\alpha-1) \eta_{j} \eta_{\imath} .
$$

In this case we say that the submanifold $M$ is $\eta$-umbilical with respect to the distinguished normal $N^{\lambda}$.

Conversely if the submanifold $M$ is $\eta$-umbilical, we can easily obtain the equations (4.20) and (4.21) by the transvection of (4.24) with $f$.

In particular, if the distinguished normal $N^{\lambda}$ to $M$ is concurrent, that is, $\nabla, N^{\lambda}=-\tau B_{\jmath}^{\lambda}$ for some function $\tau$, then we have form (4.11)

$$
\tau \delta_{\jmath}{ }^{h}=h_{\jmath}{ }^{h}, \quad \nabla_{\jmath} \nu_{p}+\nu_{q} l_{\jmath q p}=0 .
$$

Since the first of these equations is expressed as

$$
h_{j i}=\tau g_{j i},
$$

then, from (4.23), we find $\alpha=\tau$. Substituting (4.25) and $\alpha=\tau$ into (4.24), we have

$$
(\tau-1)\left(g_{j i}-\eta_{j} \eta_{\imath}\right)=0,
$$

which implies $\tau=1$. Consequently we have $h_{j i}=g_{j i}$. Thus we have

THEOREM 6. Suppose that the submanifold $M$ of codimension $l$ of a Kaehlerian manifold $\tilde{M}$ admits an almost contact metric compound structure $\left(f, g, v, f^{\perp}\right)$. In order for the almost contact metrac structure $(f, g, \xi, \eta)$ on $M$ to be normal contact, that is, Sasakian, it is necessary and sufficient that $M$ is $\eta$-umbilical with respect to the distingurshed normal $N^{\lambda}$. In addition, if the distingurshed normal $N^{2}$ to $M$ is concurrent, then $M$ is umbilical with respect to $N^{2}$.

\section{$\S 5$. Submanifolds of codimension $l$ of an even-dimensional Euclidean space}

In this section we assume that $M$ is a submanifold of codimension $l$ of an even-dimensional Euclidean space $E^{n}$ and an almost contact metric compound structure $\left(f, g, v, f^{\perp}\right)$ is induced on $M$. Then the Gauss, Codazzi and Ricci equations are given by

$$
\begin{gathered}
K_{k j i h}=h_{k h p} h_{j i p}-h_{\jmath h p} h_{k \imath p}, \\
\nabla_{k} h_{j i q}-\nabla_{j} h_{k \imath q}=-l_{\jmath q p} h_{k h p}+l_{k q p} h_{j i p}, \\
\nabla_{k} l_{j q p}-\nabla_{j} l_{k q p}=h_{\jmath q}^{l} h_{k l p}-h_{k}^{l}{ }_{q} h_{\jmath l p}+l_{k q r} l_{\jmath r p}-l_{\jmath q r} l_{k r p}
\end{gathered}
$$

respectively, where $K_{k j i}{ }^{h}=g^{h l} K_{k j \imath l}$ is the curvature tensor of $M$.

Now we shall prove 
THEOREM 7. Let $M$ be a submanifold of dimension $m>3$ in an even-dimensional Euclidean space $E^{n}$ and assume that the induced metric compound structure $\left(f, g, v, f^{\perp}\right)$ is almost contact. Then, in order for the submanifold $M$ to be umbilical with respect to the distinguished normal $N^{2}$ and $N^{2}$ parallel to the mean curvature vector of $M$ in $E^{n}$, it is necessary and sufficient that the distingurshed normal $N^{\lambda}$ is concurrent. In this case the mean curvature of $M$ is constant.

Proof. If the submanifold $M$ is umbilical with respect to the distinguished normal $N^{\lambda}$ and $N^{\lambda}$ is parallel to the mean curvature vector $H^{\lambda}$ of $M$, we have

$$
\begin{gathered}
h_{j i}=\rho g_{j i}, \\
h_{l}{ }^{l}{ }_{p}=h_{l}{ }^{l} \nu_{p}=m \rho \nu_{p}
\end{gathered}
$$

for a certain scalar function $\rho$. By means of (5.4) the equations of (4.14) and (4.12) have the following expressions

$$
\begin{gathered}
\nabla_{\jmath} \eta_{\imath}=\rho f_{j i}, \\
\nabla_{k} f_{j i}=\rho\left(\eta_{\jmath} g_{k i}-\eta_{i} g_{k \jmath}\right)
\end{gathered}
$$

respectively.

Substituting (4.15) and (5.6) into (4.13), we have

$$
\rho f_{j i} \nu_{p}+\eta_{\imath} \xi^{l} h_{\jmath l q} f_{q p}=h_{j i q} f_{q p}-f_{\imath}{ }^{l} h_{j l p},
$$

and, transvecting this equation with $g_{j i}$,

This equation implies

$$
\xi^{j} \xi^{\imath} h_{j i q} f_{q p}=h_{l}^{l} f_{q p}=0 \text { 。 }
$$

$$
\xi^{j} \xi^{2} h_{\jmath i q}=A \nu_{q},
$$

where $A=\hat{\xi}^{j} \xi^{2} h_{j i}=\rho$ and consequently

$$
\xi^{j} \xi^{\imath} h_{j i q}=\rho \nu_{q} .
$$

If we transvect (5.2) with $\nu_{q}$ and make use of (4.15), we have

$$
\nabla_{k} h_{j i}-\nabla_{\jmath} h_{k \imath}=\xi^{l} h_{k l q} h_{\jmath \imath} f_{q p}-\xi^{l} h_{j l q} h_{k \imath p} f_{q p}
$$

or, by means of (5.4),

$$
\left(\nabla_{k} \rho\right) g_{j i}-\left(\nabla_{j} \rho\right) g_{k \imath}=\xi^{l} h_{k l q} h_{j i p} f_{q p}-\xi^{l} h_{j l q} h_{k \imath p} f_{q p} .
$$

Differentiating (5.6) covariantly and using (5.7), we have

$$
\nabla_{k} \nabla_{j} \eta_{i}=\left(\nabla_{k} \rho\right) f_{j i}+\rho^{2}\left(\eta_{j} g_{k i}-\eta_{i} g_{k \jmath}\right),
$$

from which, using the Ricci identity,

$$
-K_{k j i}{ }^{h} \eta_{h}=\left(\nabla_{k} \rho\right) f_{j i}-\left(\nabla_{\jmath} \rho\right) f_{k i}+\rho^{2}\left(\eta_{\jmath} g_{k i}-\eta_{k} g_{\jmath \imath}\right) .
$$


From this, by the Bianchi identity, we obtain

$$
\left(\nabla_{k} \rho\right) f_{j i}+\left(\nabla_{j} \rho\right) f_{\imath k}+\left(\nabla_{\imath} \rho\right) f_{k j}=0 .
$$

Transvecting (5.12) with $f^{j i}$, we get

$$
(m-3) \nabla_{k} \rho+2 \xi^{l}\left(\nabla_{l} \rho\right) \eta_{k}=0 .
$$

Moreover the transvection of (5.12) with $\xi^{\imath} f^{k \jmath}$ yields $\xi^{l} \nabla_{l} \rho=0$. Therefore we see that $\rho$ is constant for $m>3$.

From (5.11) and the above result we have

$$
\xi^{l} h_{k l q} h_{j i p} f_{q p}=\xi^{l} h_{j l q} h_{k \imath p} f_{q p},
$$

and, transvecting with $\xi^{\jmath}$ and using (5.9),

$$
\xi^{l} h_{k l q} \xi^{\jmath} h_{j i p} f_{q p}=0 \text {. }
$$

Transvecting (5.8) with $\xi^{\jmath} \xi^{k} h_{k h p}$ and using (5.13), we have

$$
f_{\imath}{ }^{l} \xi^{j} h_{j l p} \xi^{k} h_{k h p}=0
$$

and, transvecting with $f_{m}^{2}$ and using (5.9),

$$
\xi^{l} h_{l \jmath p} \xi^{k} h_{k \imath p}=\rho^{2} \eta_{j} \eta_{\imath} .
$$

Let $H$ be the matrix $\left(\xi^{l} h_{l \jmath p}\right)$. Then (5.14) means that ${ }^{t} H H=\rho^{2}\left(\eta_{j} \eta_{\imath}\right)$, where ${ }^{t} H$ is the transpose of $H$. Since the rank of matrix $\left(\eta_{j} \eta_{\imath}\right)$ is 1 , then the rank of $H$ is also 1 . Therefore we may put

$$
\xi^{l} h_{l \imath p}=\rho \eta_{i}^{\prime} \nu_{p}^{\prime} \text {. }
$$

Comparing the transvection of (5.15) with $\xi^{\imath}$ and (5.9), we see that $\nu_{p}=A \nu_{p}^{\prime}$, where $A=\xi^{l} \eta_{l}^{\prime}$. Hence we have

$$
\xi^{l} h_{l \imath p} f_{p q}=0
$$

or equivalently, from (4.15),

$$
\nabla_{j} \nu_{q}+\nu_{p} l_{j p q}=0
$$

Finally we see, from (5.6) and (5.17), that the distinguished normal $N^{2}$ is concurrent.

Conversely if the distinguished normal $N^{\lambda}$ is concurrent, that is, $\nabla_{j} N^{\lambda}=$ $-\tau B_{j}{ }^{\lambda}$ for a certain function $\tau$, then we have $h_{j i}=\tau g_{j i}$, which shows that $M$ is umbilical with respect to $N^{\lambda}$, and (5.17). Substituting (4.14) and the above equations into (4.13), we have

$$
f_{\imath}{ }^{l} h_{j l p}=h_{j i q} f_{q p}-\tau f_{j i} \nu_{p},
$$

and, transvecting with $g^{j i}$, 
which implies

$$
h_{l}^{l} f_{q p}=0
$$

$$
h_{l}{ }^{l}=h_{l}{ }^{l} \nu_{q}=m \tau \nu_{q} .
$$

Therefore the distinguished normal $N^{\lambda}$ is parallel to the mean curvature vector $H^{\lambda}$.

In this case we easily see that the mean curvature of $M$ is constant. This completes the proof.

Now we assume that the mean curvature vector $H^{\lambda}$ is parallel to the distinguished normal $N^{\lambda}$ of $M$, that is, $H^{\lambda}=\rho N^{\lambda}$ for a certain function $\rho$. Then we have (5.5).

If the submanifold $M$ is pseudo-umbilical, we have

$$
G_{\lambda \mu} h_{\jmath i}{ }^{\lambda} H^{\mu}=\rho^{2} g_{\jmath i}
$$

because $|\rho|$ is the length of $H^{\lambda}$. From (5.5) and (5.18) we find that $h_{j i}=|\rho| g_{j i}$, which means that $M$ is umbilical with respect to the distinguished normal $N^{\lambda}$.

Conversely if the submanifold $M$ is umbilical with respect to $N^{\lambda}$, we have (5.18) from (5.4) and (5.5). Thus we have

THEOREM 8. Let $M$ be a submanifold of codimension $l$ with the induced almost contact metric compound structure $\left(f, g, v, f^{\perp}\right)$ of an even-dimensional Euclidean space $E^{n}$ and the mean curvature vector $H^{\lambda}$ of $M$ parallel to the distinguished normal $N^{\lambda}$ of $M$ in $E^{n}$. Then, in order for the submanfold $M$ to be pseudo-umbilical, it is necessary and sufficient that $M$ is umbilical with respect to the distınguished normal $N^{2}$.

It is well-known that pseudo-umbilical submanifolds in a Euclidean space with the mean curvature vector parallel in the normal bundle are minimal submanifolds of a hypersphere [7]. From Theorem 7, we see that the mean curvature vector is parallel in the normal bundle. Therefore it follows from Theorems 7 and 8 that the submanifold $M$ of dimension $m>3$ is contained as a minimal submanifold in a hypersphere in $E^{n}$.

On the other hand, we see that the direct sum of the tangent space of $M$ and the distinguished normal $N^{\lambda}$ is invariant because of (4.3) and (4.5). Therefore $M$ is an intersection of a complex cone with generator $N^{\lambda}$ on $M$ and an $(n-1)$-dimensional sphere.

Thus we have the following

THEOREM 9. Let $M$ be a submanifold of codimension $l$ with the induced almost contact metric compound structure $\left(f, g, v, f^{\perp}\right)$ of an even-dimensional Euclidean space $E^{n}$. If the submanifold $M$ satisfies one of the followings;

(1) $M$ of dimension $m>3$ is umbilical with respect to the distingurshed normal $N^{\lambda}$, and $N^{\lambda}$ parallel to the mean curvature vector,

(2) $M$ of dimension $m>3$ is pseudo-umbilical submanifold and the distıngurshed 
normal $N^{i}$ parallel to the mean curvature vector,

(3) The distinguished normal $N^{\lambda}$ is concurrent,

then $M$ is the intersection of a complex cone with generator $N^{\lambda}$ and an $(n-1)$-dimensional sphere.

We now assume that the metric compound structure $\left(f, g, v, f^{\perp}\right)$ induced on a submanifold $M$ of codimension $l$ of an even-dimensional Euclidean space $E^{n}$ defines a normal almost contact metric structure $(f, g, \xi, \eta)$ on $M$ and the distinguished normal $N^{\lambda}$ is parallel in the normal bundle of $M$. Then we have the equation (4.20), that is,

$$
h_{j l} f_{\imath}{ }^{l}+h_{\imath l} f_{\jmath}{ }^{l}=0 .
$$

Transvecting (5.19) with $f_{k}{ }^{2}$ and taking the skew-symmetric part, we have

$$
h_{j l} \xi^{l} \eta_{k}=h_{k l} \xi^{l} \eta_{\jmath},
$$

which means that we may put

$$
h_{j l} \xi^{l}=\alpha \eta_{\jmath},
$$

where $\alpha=\xi^{\jmath} \xi^{2} h_{j i}$. Differentiating (5.20) covariantly and substituting (4.14) into this equation, we have

$$
\left(\nabla_{k} h_{j l}\right) \xi^{l}+h_{\jmath}{ }^{l}\left(-h_{k \imath} f_{l}{ }^{i}\right)=\left(\nabla_{k} \alpha\right) \eta_{\jmath}+\alpha\left(-h_{k l} f_{\jmath}{ }^{l}\right)
$$

and, taking the skew-symmetric part and using (5.19), the equation

$$
\left(\nabla_{k} h_{j l}-\nabla_{j} h_{k l}\right) \xi^{l}+2 h_{j}{ }^{l} h_{l \imath} f_{k}{ }^{2}=\left(\nabla_{k} \alpha\right) \eta_{j}-\left(\nabla_{j} \alpha\right) \eta_{k}+2 \alpha h_{j l} f_{k}{ }^{l} .
$$

On the other hand, since $N^{\lambda}$ is parallel in the normal bundle of $M$, we have (5.17) or equivalently (5.16). From (5.10) and (5.16) we find

$$
\nabla_{k} h_{j i}-\nabla_{j} h_{k i}=0 \text {. }
$$

Substituting (5.22) into (5.21), we have

$$
2 h_{\jmath}{ }^{l} h_{l \imath} f_{k}{ }^{2}=\left(\nabla_{k} \alpha\right) \eta_{\jmath}-\left(\nabla_{\jmath} \alpha\right) \eta_{k}+2 \alpha h_{j l} f_{k}{ }^{l},
$$

and transvecting (5.23) with $\xi^{2}$ and using (5.20),

$$
\nabla_{k} \alpha=A \eta_{k}
$$

where $A=\xi^{l} \nabla_{l} \alpha$. Thus (5.23) implies

$$
h_{\jmath}{ }^{l} h_{l \imath} f_{k}{ }^{2}=\alpha h_{j l} f_{k}{ }^{l} .
$$

If we transvect this equation with $f_{h}{ }^{k}$ and make use of (5.20), we obtain

$$
h_{\jmath}{ }^{l} h_{l \imath}=\alpha h_{\jmath \imath} \text {. }
$$

Differentiating (5.24) covariantly and substituting (4.14) into this equation, we 
have

$$
\left(\nabla_{k} A\right) \eta_{j}-\left(\nabla_{\jmath} A\right) \eta_{k}+2 A h_{\jmath l} f_{k}^{l}=0,
$$

and, transvecting with $\xi^{j}$ and using (5.19),

$$
\nabla_{k} A=\left(\xi^{l} \nabla_{l} A\right) \eta_{k} .
$$

The two equations above show that $A h_{\jmath} f_{k}{ }^{l}=0$. Transvecting this equation with $f_{2}{ }^{k}$ and using (5.20), we have

$$
A\left(h_{j i}-\alpha \eta_{j} \eta_{2}\right)=0 .
$$

Now suppose that $M$ is locally irreducible. Then we have $A=0$ from (5.26). In fact, if $A \neq 0$, we have $h_{j i}=\alpha \eta_{j} \eta_{\imath}$. Substituting this equation into (4.14), we find $\nabla_{j} \eta_{2}=0$, which means that $\xi^{h}$ is parallel vector field. This contradicts to the local irreducibility of $M$. Therefore we see that $\alpha$ is constant from (5.24). Moreover this constant is nonzero. In fact, if $\alpha=0$, we have $h_{j 2}=0$ from (5.25) and finally we also have $\nabla_{j} \eta_{\imath}=0$.

Differentiating (5.25) covariantly, we have

$$
\left(\nabla_{k} h_{j l}\right) h_{\imath}{ }^{l}+h_{\jmath}{ }^{l}\left(\nabla_{k} h_{i l}\right)=\alpha \nabla_{k} h_{j i} .
$$

From this equation, taking the skew-symmetric part with respect to $\imath$ and $k$ and using (5.22), we have

$$
\left(\nabla_{k} h_{j l}\right) h_{\imath}{ }^{l}-\left(\nabla_{i} h_{j l}\right) h_{k}{ }^{l}=0 .
$$

Since the sum of this equation and one with exchanged $\jmath$ and $k$ is

$$
2\left(\nabla_{k} h_{j l}\right) h_{\imath}{ }^{l}=\alpha \nabla_{k} h_{j i}
$$

by means of (5.22), then we have, transvecting (5.27) with $h_{h}{ }^{2}$ and using (5.25) and $\alpha \neq 0$,

$$
\left(\nabla_{k} h_{\jmath l}\right) h_{\imath}{ }^{l}=0 .
$$

Therefore, from (5.27) and (5.28), we have

$$
\nabla_{k} h_{j i}=0 \text {. }
$$

By the irreducibility of $M$, it follows from (5.29) that $h_{j i}$ is proportional to $g_{j i}$ and from (5.25) that the proportional factor is equal to $\alpha$, that is,

$$
h_{j i}=\alpha g_{j i} .
$$

Consequently we see, from (5.17) and (5.30), that the distinguished normal $N^{\lambda}$ is concurrent.

Thus, from Theorem 9, we have

THEOREM 10. Let $M$ be a locally irreducible submanfold of codimension $l$ 
with an induced metric compound structure $\left(f, g, v, f^{\perp}\right)$ of a Euclidean space $E^{n}$ such that the distingurshed normal $N^{\lambda}$ is parallel in the normal bundle. If the metric compound structure $\left(f, g, v, f^{\perp}\right)$ defines a normal almost contact metric structure $(f, g, \xi, \eta)$ on $M$, then $M$ is the intersection of a complex cone with generator $N^{\lambda}$ and an $(n-1)$-dimensional sphere.

\section{§6. Metric compound structure $\left(f, g, v, f^{\perp}\right)$ in which $f^{\perp}=0$}

Let the set $\left(f, g, v, f^{\perp}\right)$ be a metric compound structure on $M$ and assume that the tensor field $f^{\perp}$ on $R^{l}$ vanishes identically. Then, from (1.14), (1.15) and (1.16), we have

$$
\begin{gathered}
f_{\jmath}{ }^{2} v_{p \imath}=0, \quad v_{q}^{\imath} f_{\imath}{ }^{n}=0, \\
v_{q}{ }^{2} v_{p \imath}=\delta_{q p} .
\end{gathered}
$$

We assume that $M$ is odd-dimensional and put $l=2 a+1$.

We choose one of the $l$ vector fields $v_{p \imath}$ as $\eta_{\imath}$, for example,

$$
\eta_{\imath}=v_{2 a+1, \imath}
$$

and put $\xi^{h}=g^{i n} \eta_{2}$. Then, by means of (6.2), we have

$$
\xi^{2} \eta_{2}=1 \text {. }
$$

Now we put

$$
\phi_{i}{ }^{h}=f_{\imath}{ }^{h}-\left(\sum_{p=1}^{a} v_{p i} v_{p}{ }^{h}-\sum_{p=1}^{a} v_{\bar{p} i} v_{p}{ }^{h}\right),
$$

where $\bar{p}=a+p$. Then, using (6.1) and (6.2), we have

$$
\dot{\phi}_{\jmath}{ }^{2} \phi_{i}{ }^{h}=f_{\jmath}{ }^{2} f_{\imath}{ }^{h}-\sum_{p=1}^{2 a} v_{p \jmath} v_{p}{ }^{h}=f_{\jmath}{ }^{\imath} f_{\imath}{ }^{h}-v_{p j} v_{p}{ }^{h}+\eta_{j} \xi^{h},
$$

which implies, from (1.13),

$$
\phi_{\jmath}{ }^{2} \phi_{2}{ }^{h}=-\delta_{\jmath}{ }^{h}+\eta \xi{ }^{h} .
$$

From (6.3) and (6.5) we also have

$$
\phi_{\jmath}{ }^{2} \xi^{\jmath}=\phi_{\jmath}{ }^{2} \eta_{\imath}=0 \text {. }
$$

Using (6.1) and (6.2), we also have

$$
\phi_{j}{ }^{k} \phi_{i}{ }^{h} g_{k h}=f_{j}{ }^{k} f_{\imath}{ }^{h} g_{k h}+v_{p j} v_{p i}-\eta_{j} \eta_{\imath},
$$

which implies, from (1.17),

$$
\phi_{j}{ }^{k} \phi_{i}{ }^{h} g_{k h}=g_{j i}-\eta_{j} \eta_{\imath} .
$$


Thus we have the following

Theorem 11. Let $\left(f, g, v, f^{\perp}\right)$ be a metric compound structure on an odddimensional manfold $M$. If the tensor $f^{\perp}$ on $R^{l}$ vanushes identically, then the manifold $M$ admits an almost contact metric structure $(\phi, g, \xi, \eta)$, where $\eta$ is one of $l$ vector fields $v$ and $\phi$ is given by (6.5).

\section{REFERENCES}

[1] B.-Y. Chen, Geometry of submanifolds. Marcel Dekker, Inc. New York (1973).

[2] B.-Y. Chen AND K. YANo, Pseudo-umbilical submanifolds in a Riemannian manifold of constant curvature. Diff. Geom. in honor of K. Yano, Kinokuniya Tokyo (1972), 61-71.

[3] S. Ishihara AND U.-H. KI, Complete Riemannian manifolds with $(f, g, u, v, \lambda)$ -structure. J· Diff. Geom. 8 (1973), 541-554.

[4] S. SASAKI, On differentiable manifolds with certain structures which are closely related to almost contact structure I; II. Tôhoku Math. J. 12 (1960), 459 $-476 ; 13$ (1961), 281-294.

[5] Y. TASHIRO, On certain structure of hypersurfaces in complex manifolds I; II. Tôhoku Math. J. 15 (1963), 62-78; 167-175.

[6] Y. TAshiro, On relations between almost complex spaces and almost contact spaces (in Japanese). Sugaku 16 (1964-1965), 54-61.

[7] K. YANO AND B.-Y. CHEN, Minimal submanifolds of a higher dimensional sphere. Tensor, N.S. 22 (1971), 369-373.

[8] K. YANO AND U.-H. KI, On $(f, g, u, v, w, \lambda, \mu, v)$-structures satisfying $\lambda^{2}+\mu^{2}+\nu^{2}=1$. Kōdai Math. Sem. Rep. 29 (1978), 285-307.

\section{HiRoshima UNIVERSITY.}

